\title{
Using Evolutionary Algorithms to Plan Automatic Minehunting Operations
}

\author{
Nuno Abreu ${ }^{1,2}$, Aníbal Matos ${ }^{1,2}$ \\ ${ }^{1}$ INESC TEC, Campus da FEUP, Rua Dr. Roberto Frias, 378, 4200-465 Porto, Portugal \\ ${ }^{2}$ FEUP - DEEC, Rua Dr. Roberto Frias, s/n, 4200-465 Porto, Portugal \\ \{nabreu,anibal.matos\}@inescporto.pt
}

\begin{abstract}
Keywords: Evolutionary Algorithms, Neural Networks, Mission Planning, AUV, 3D Coverage, Minehunting.
Abstract: While autonomous underwater vehicles (AUVs) are increasingly being used to perform mine countermeasures (MCM) operations, the capability of these systems is limited by the efficiency of the planning process. In this paper we study the problem of multiobjective MCM mission planning with an AUV. In order to overcome the inherent complexity of the problem, a multi-stage algorithm is proposed and evaluated. Our algorithm combines an evolutionary algorithm (EA) with a local search procedure based on simulated annealing (SA), aiming at a more flexible and effective exploration and exploitation of the search space. An artificial neural network (ANN) model was also integrated in the evolutionary procedure to guide the search. The results show that the proposed strategy can efficiently identify a higher quality solution set and solve the mission planning problem.
\end{abstract}

\section{INTRODUCTION}

AUVs are able to perform underwater minehunting missions with higher efficiency, reduced search time and even with covert search capability when compared to traditional methods. In order to quickly and safely demine an area, the AUV needs to properly navigate through that area. Hence, a path needs to be carefully planned so all the region is sampled. There is an abundance of path planning algorithms in literature and, for this specific application, a small subset known as coverage path planning algorithms is usually used (Choset, 2001).

Solving a multiobjective path planning problem is computationally very expensive. EAs have been successfully used in the past for solving the multiobjective path planning problem (Fujimura, 1996; Xiao et al., 1997). This approach is an alternative to classical optimization methods with the capability of solving problems involving a big search space quickly, although not guaranteeing that an optimal solution is found.

This article consists of four sections and is structured as follows. Section 2 presents our multiobjective mission planning problem and section 3 presents an EA designed to solve it. Section 4 presents and discusses the obtained results. Finally, in the last section we give some conclusions and ideas for future work.

\section{MISSION PLANNING}

The most popular approach used for underwater mine hunting is performing complete coverage of the operational area with the robot's sensors. When acquiring data with a sonar-equipped vehicle, a lawnmowing search pattern (Land and Choset, 1998) with several parallel tracks is standard if no prior information on potential target locations is available.

In the underwater domain, coverage path planning research has its most important application in the context of MCM applications. (Stack and Smith, 2003) presented a 2D coverage algorithm for MCM using cell decomposition. They investigate a planning scheme for incomplete coverage, as it may not be feasible in terms of time and energy costs to execute complete coverage. This scheme divides the search area into cells and surveys each cell using a conventional line-sweep pattern with a row spacing that is larger than the sensor footprint, exploring the fact that mines are normally placed in lines. They assume that if mines are evenly spaced, then randomly varying the spacing between each row in the lawn-mowing pattern will decrease the probability of missing an entire mine line. An acceptable probability of detection (POD) is ensured by establishing bounds on row spacing. A perfect POD is assumed for any mine within the sensor footprint. 
(Fang and Anstee, 2010) developed an algorithm for 3D offline mission planning, combining Boustrophedon decomposition with the generalized Voronoi diagram, to cover a well-known planar seabed using an AUV fitted with side-looking sonar. The vehicle can navigate over terrain at constant altitude. They consider both even and uneven lawn-mowing coverage patterns but the spacing is fixed, proportional to range setting. Since they assume a planar seabed, they do not consider a complex topography and therefore there was no need to implement variable spacing between the segments.

(Williams, 2010) presented an 2D offline coverage algorithm for MCM that optimizes the spacing between parallel tracks in order to maximize POD, considering seabed type and range. Their track-spacing algorithm consists of an exhaustive greedy search for the best tracks. To further improve these selected tracks a small geographical displacement of each track is considered. The main disadvantage of this greedy algorithm is that solutions are only locally optimal.

\subsection{Measures of Mission Performance}

In order to assess the effectiveness of a MCM operation we need to be able to estimate the detection performance that should be achieved in a specific mission.

\subsubsection{Lateral Range}

The concept of lateral range curve (LRC) was introduced by (Koopman, 1999). Imagine a searcher following an infinitely long, straight path, searching on either side of that path. Lateral range refers to the perpendicular distance an object is to the searcher's path. That searcher's LRC $p(x)$ is the probability of detecting a stationary object that is at its closest exactly a distance $x$ from the searcher's path. The LRC is derived experimentally by moving a sensor through an area, where objects are randomly placed (the positions are known), using parallel straight-line search transects and then testing the detection system.

\subsubsection{Probability of detection}

POD is an estimate of how likely it will be for a search performed in a given area to find an object, assuming it is there. It is known that an overlap in area coverage can improve the detection performance. When it is assumed that multiple searches are executed independently of each other, the combined POD is given by:

$$
\text { PODcum }=1-\prod_{i=1}^{n}\left(1-P_{i}\right)
$$

If assuming complete correlation, the combined POD is simply the higher of the two probabilities. When there is an indeterminate amount of correlation an accepted practice (Nash et al., 1982) is to average the probabilities obtained assuming complete independence. Given the risk involved in these operations, it is advisable to obtain conservative estimates by assuming complete correlation between searches.

\subsection{Covering With Different Sensors}

The sidescan sonar uses one transducer on each side to emit acoustic beams down towards the seafloor, across a wide angle perpendicular to the path of the sensor through the water, and receives the echos of those beams. All sidescan sonars suffer from an inability to illuminate targets within the "nadir-gap" area (section below the transducers).

The multibeam bathymetric sonar uses two or more perpendicular transducer arrays to transmit and receive the beams. Each received beam allows the estimation of the range (calculated from the time delay) to the seafloor. By combining data from consecutive pings (range and angle of the received beam), a 3D map of the seafloor can be generated.

The most relevant feature to consider in mission planning is the existence of the nadir gap in sonar coverage, as illustrated in figure 1 . The traditional approach to compensate for this feature is to partially overlap pairs of swaths so their nadir gaps are covered, also known as uneven lawn-mowing.

\subsection{Problem Statement}

The principal problem under study in this paper is how to design and implement a more flexible 3D path planning algorithm that enables an AUV to efficiently cover the bottom of a submerged area with no missed areas and with a specified minimum POD. The planner should identify a set of parallel transects, representing sonar swaths, that maximize the estimated performance of a MCM operation, using the available knowledge and resources.

\subsubsection{Decision variables}

- Mission path

A mission path is represented by a set of consecutive straight-line tracks, also referred as swaths (data ac- 


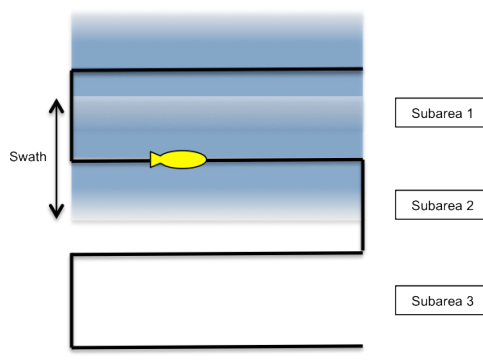

(a) Coverage with a multibeam sonar

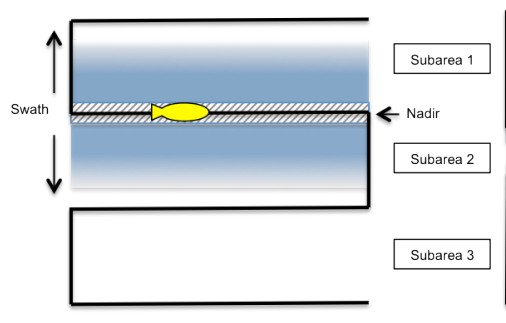

(b) Coverage with a sidescan sonar

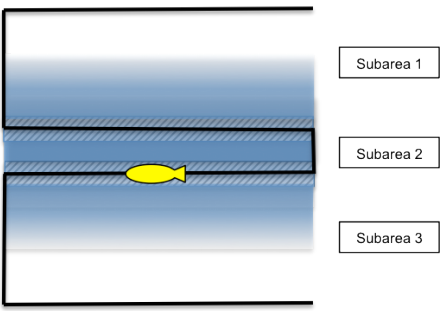

(c) Uneven lawn-mowing technique

Figure 1: Different methodologies used in coverage problems.

quired by the sonar):

$$
P=\left\{s_{1}, s_{2}, \ldots, s_{n}\right\}
$$

- Vehicle Velocity

The AUV will follow the specified path with a constant forward velocity relative to water equal to $v_{\text {water }}$.

\subsubsection{Objectives}

- Maximize probability of detection

The MCM effectiveness is maximized by maximizing the POD of the target on the survey area:

$$
\max \sum \overline{P O D_{s}}(S, P)
$$

- Minimize energy consumption

The amount of energy consumed by the vehicle depends on its velocity, orientation and the actual path.

$$
\min E\left(P, v_{\text {water }}\right)
$$

- Minimize time to complete the mission

The time required to execute the mission depends only on the path and the component of velocity that is parallel to the track direction.

$$
\min T\left(P, v_{\text {earth }}\right)
$$

\subsubsection{Constraints}

Searching for a path requires the consideration of the terrain topography, vehicle constraints (battery capacity, maximum velocity and maximum steering angle), sensor constraints (sonar maximum range and vertical beam angle) and mission contraints (maximum time available for the mission and minimum global POD).

\section{METHODS}

\subsection{Multiobjective Problems}

In a multiobjective optimization problem, the goal is to find a set of different solutions representing distinct trade-offs among the different objectives. These solutions are known as Pareto-optimal solutions (Deb, 2001). This set of solutions can be found using the Pareto Optimality Theory (Deb, 2001).

The continuous improvement in hardware technology has allowed the use of algorithms with higher complexity such as EAs, providing efficient means for addressing real world problems that traditional algorithms were unable to conquer. These techniques are based on the principles of natural evolution as presented by (Darwin, 1859). Pareto dominancebased techniques remain the most popular selection scheme adopted by multiobjective evolutionary algorithms (MOEAs), because of the several advantages that it provides. The SPEA 2 (Zitzler et al., 2001) is one of the most popular MOEAs used when comparing a newly designed MOEA.

\subsection{Proposed EAs for Mission Planning}

Our approach is a a posteriori technique, more specifically a Pareto-based approach. The mission planning algorithm is an off-line planner that should be executed before the vehicle is deployed in the target area.

\subsubsection{Individual Representation}

We consider an individual as a solution represented by a vector of real valued parameters $x \in R_{n}$. Thus, an individual is represented by:

$$
\text { Ind } \in(t, \theta, d, a, v)
$$

where $t$ is the track spacing, $\theta$ is the track direction, $d$ is the depth of the path (when planning with constant depth), $a$ is the altitude of the path relative to the seafloor (when planning with constant altitude) and $v$ is the vehicle velocity.

\subsubsection{Informed EA}

Our approach combines an EA with an approximation technique, more specifically ANNs. EAs usually need 
a sufficiently large number of function evaluations to reach the optima of the problem. The approximate model is used to reduce the number of expensive exact function evaluations. The main task of the approximate model is to capture the general trend of the fitness landscape and guide the search towards the better regions while reducing the number of exact function evaluations needed to find the best solutions. This guidance is executed by assessing the performance of multiple candidate individuals using the approximate models, with a much smaller evaluation time, and choosing only the best to integrate the next generation population. One of the challenges that arises is the need to update the approximate model using new information from exact function evaluations throughout the execution of the algorithm, adding finer details to the fitness landscape.

\section{Initialisation}

- Randomly generates $N_{p}$ individuals from a uniform distribution in decision space;

- Uses them to train the approximate model.

\section{Neural Network Training}

- Uses a classical multilayer feedforward ANN using the standard error back-propagation algorithm;

- The ANN receives in the input six parameters that define an individual, and generates the estimated objective function values at the output;

- Executed every $n_{t}$ generations;

- Since it is proven (Huang, 2003) that a ANN with two hidden layers can approximate any function with negligibly small error, we test networks with different number of neurons in each layer and choose the best topology (smaller mean square error in cross-validation).

\section{Fitness Assignment}

- Same procedure as in SPEA2.

\section{Environmental Selection}

- Uses variable archive and population sizes (avoids creating clusters of individuals);

- Controls the individual's density in the containers.

\section{Termination}

- The EA stops when a pre-specified number of generations is achieved, when the maximum execution time is achieved or when it converges.

\section{Mating Selection}

- Mating occurs between individuals in the archive and between individuals in the archive and the population;

- The pair should be close to each other in objective space to minimize the randomization effect.

\section{Recombination}

- Evaluates candidate offspring using the approximate model (cheaper);

- Ranks them in terms of improvement over the less fit parent (choosing one objective function);

- Evaluates the best one using the exact objective functions and adds it to the population;

- Should significantly speed up the EA because we can quickly test multiple candidate individuals.

\section{Mutation}

- Evaluates possible mutations using the approximate model (cheaper);

- Performs sensitivity analysis using the ANN, testing different variations of the original individual and analysing the estimated performance;

- Ranks them in terms of improvement over the original individual;

- Evaluates the best one using the exact objective functions and adds it to the population.

\subsection{Local Optimization}

Our local search stage, based on SA, has been designed to optimize two distinct objectives, namely:

- Minimize the uncovered area;

- Maximize the average POD.

This is a combinatorial problem where we have to choose the best set of inter track distances maximizing the coverage of an area characterized by a specific topography. We use distinct strategies depending on the type of sonar that is being used for seafloor mapping, more specifically if using multibeam (no nadir gap) or sidescan sonar (with nadir gap).

\section{Strategy for the Case Without Nadir Gap}

\section{Initialization}

- Determines the original spacing between tracks (kept constant by the EA);

- Neighbourhood size is set to the initial spacing. 


\section{Evaluation}

- Determines the insufficiently covered area percentage and the average POD (per subarea and total as seen in Figure 1);

- Accept the solution if better then the last or apply the Metropolis criterion (Metropolis et al., 1953).

\section{Check for termination}

- Terminates when the temperature reaches a minimum value or when it converges.

\section{Mutation}

- Selects the subarea between a pair of tracks with lower coverage quality;

- Places two tracks closer together, achieving higher coverage in the subarea in between;

- The amplitude of the mutation $\Delta_{i}$ is obtained from equation 8 , where $r$ is a random number between -1 and $1, N$ is the neighborhood size and $T$ is the temperature of the process. It must be inferior to the previous track spacing $s_{i}$ in subarea $i$;

- Update spacing $s_{i}$ (equation 7);

- Calculate the contribution (weights $w_{j}$ ) of each subarea $\Delta_{j}(j \neq i)$ to compensate for $\Delta_{i}$ (equations 12 and 10$)$

- If there are no uncovered areas (ucells $=0)$ then maximize the average POD (avgpod) using a similar procedure where track spacing is decreased in the region where the average POD is lower;

- In this case the contribution of each region $\Delta_{j}$ depends directly on the average POD in each of those regions (equation 11).

$$
\begin{gathered}
s_{i}=s_{i}-\Delta_{i} \\
\Delta_{i}=r N T \\
\Delta_{i}=-\sum_{\substack{j=1 \\
j \neq i}}^{k} \Delta_{j} \\
w_{j}^{\prime}=\frac{1}{u c e l l s_{j}} \\
w_{j}^{\prime}=\operatorname{avgpod}_{j} \\
w_{j}=\frac{w_{j}^{\prime}}{\sum_{\substack{j=1 \\
j \neq i}}^{k} w_{j}^{\prime}} \\
\Delta_{j}=w_{j} \times \Delta_{i}
\end{gathered}
$$

\section{Strategy for the Case With Nadir Gap}

The most used technique to handle this problem is the uneven lawn-mowing coverage pattern, where consecutive pairs of tracks cover each other's nadir. Here we make the distinction between odd and even areas because they are analysed differently. Even areas will have smaller spacing since the adjacent tracks need to be closer together in order to cover their nadir gaps. This strategy differs from the previous one on the mutation phase:

\section{Mutation}

- Similar to the previous one, except that adjusting odd subareas only affects other odd subareas, while adjusting even subareas only affects adjacent subareas.

- The purpose of controlling track spacing in even subareas is mainly to cover their nadir gaps, therefore only the adjacent subareas should compensate for the variation of track spacing.

- Even subareas should remain untouched when adjusting odd subareas so the nadir gaps remain covered.

\section{RESULTS}

With the following experiments we intend to:

- demonstrate that our multi-stage multiobjective algorithm can solve the mission planning problem successfully;

- show that the integration of our local search strategy increases the efficiency of the search process.

\subsection{Standard VS Informed EA}

This experiment aims at proving the advantage of integrating the ANN in the EA. Table 1 shows the obtained results. We can observe that at the initial stages the Pareto set obtained by the informed EA is dominated to a larger extent by the one obtained by the standard EA (no ANN). This can be due to the longer initialization phase that is executed on the former, as the ANN needs to be trained, while the latter starts the evolutionary process sooner. The increasing dominance that the Pareto set generated by the informed EA exhibits over time demonstrates that the ANN is guiding the search to more promising regions, increasing speed of convergence. 
Table 1: Comparison of the obtained non-dominated sets as the EAs are executed.

\begin{tabular}{|c|c|c|c|c|c|c|c|c|}
\hline Time (min) & \multicolumn{2}{|c|}{10} & \multicolumn{2}{c|}{20} & \multicolumn{2}{c|}{40} & \multicolumn{2}{c|}{60} \\
\hline EA & Std & Inf & Std & Inf & Std & Inf & Std & Inf \\
\hline \% Dominated & 2.70 & 26.67 & 10.11 & 18.60 & 23.71 & 12.50 & 30.39 & 4.76 \\
\hline Generations & 6 & 4 & 15 & 14 & 26 & 31 & 37 & 50 \\
\hline
\end{tabular}

Table 2: Design constraints used in this test.

\begin{tabular}{|c|c|}
\hline Parameter & Bounds \\
\hline Battery capacity & $800 \mathrm{Wh}$ \\
\hline Velocity relative to water & $1 \leq v \leq 3$ \\
\hline Sonar maximum range & $80 \mathrm{~m}$ \\
\hline Sonar vertical beam angle & $10 \leq \alpha \leq 70$ \\
\hline Maximum operating time & $10 \mathrm{~h}$ \\
\hline
\end{tabular}

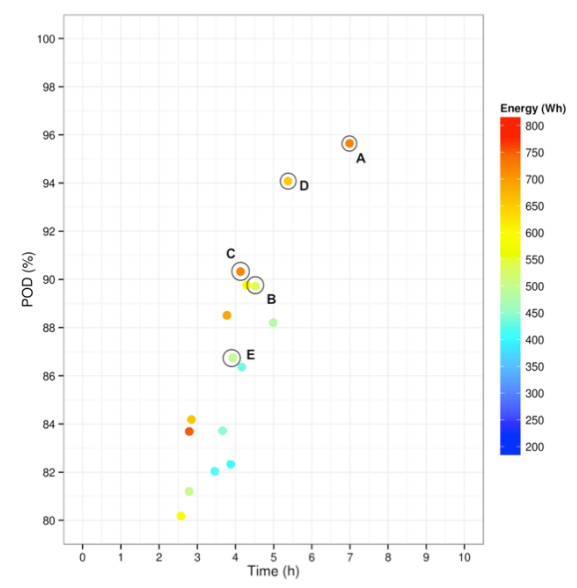

Figure 2: Example of a Pareto Front containing several solutions for the mission planning problem.

\subsection{Mission Planning With EAs}

It is important to exemplify what will be the typical output of the execution of our planning algorithm. We decided to stop the algorithm after 1 hour so we could analyse its behaviour in detail. Our planning problem was subject to the constraints detailed in table 2 . We selected 5 distinct solutions from the Pareto front shown in figure 2 and these are fully described in table 3 . They were chosen because they clearly demonstrate the trade-offs between the objective function values. We can observe that a higher detection performance implies more time to complete the mission. As expected, we can also observe that the detection performance is better when using smaller track spacing. The velocity also has considerable impact on performance as the best solutions (in terms of detection performance) use the minimum values for this variable as the energy that is saved by doing so is being used to increase the length of the path.

\subsection{Local Optimization}

Non-dominated solutions previously found by our informed EA were used as initial solutions here. Table 4 presents descriptive statistics for the optimized set of solutions for covering a given area with a sidescan sonar. It shows the variation in the amount of insufficiently covered cells ("MinRatio") and the variation in POD. In average we obtained approximately a decrease of $5 \%$ in the former and a increase of $0.6 \%$ in the latter, clearly showing the improvement made by local search in this scenario. This demonstrates the need for uneven lawn-mowing coverage pattern when using a sidescan sonar. The obtained results allowed us to take some additional conclusions related to the usefulness of this search process. As explained earlier, it is required an even number of tracks to cover the nadir gaps caused by the use of a sidescan sonar. If the original number of tracks is odd, then we need to add or remove a track in order to use our algorithm. The simulations showed that while the performance is positively affected by an addition of a track, it is negatively affected by its removal. The local optimization algorithm could not compensate the decrease in performance caused by the removal of a track. Table 5 presents the descriptive statistics for the optimized set of solutions excluding the ones where tracks were removed. It can be seen that the average POD increases and that the amount of cells with insufficient coverage is reduced. In this scenario the preferred action is to add a track, but since mission planing constraints need to be respected, it may not be possible to do so. Therefore we conclude that, in a time critical application such as the one being addressed here, it may be better to simply skip the local optimization of solutions that required removal of a track since it is not worth the extra computational time. Figure 4 shows a graphical representation of a given solution before and after the local optimization procedure was executed. The improvement in this case was a decrease of $14.0 \%$ in the amount of insufficiently covered cells and an increase of $0.9 \%$ in the average POD. This is visually identified by the almost inexistence of red regions in the optimized solution plot. Notice that the tracks were simply rearranged maintaining its direction and depth and that no tracks were added or removed. This demonstrates the usefulness and com- 
Table 3: Some distinct solutions in the Pareto Front.

\begin{tabular}{|c|c|c|c|c|c|c|c|c|}
\hline s & $\overline{P O D}(\%)$ & Energy (Wh) & Time (h:m:s) & Depth $(\mathrm{m})$ & Altitude $(\mathrm{m})$ & Spacing $(\mathrm{m})$ & $\mathrm{V}$ & Dir (o) \\
\hline A & 95.65 & 728.80 & $6: 59: 20$ & - & 13.24 & 41.13 & 1.07 & 91.44 \\
\hline B & 89.72 & 537.04 & $4: 31: 11$ & - & 11.63 & 63.26 & 1.14 & 32.31 \\
\hline C & 90.33 & 723.73 & $4: 07: 59$ & 82.38 & - & 57.46 & 1.34 & 259.87 \\
\hline D & 94.08 & 656.93 & $5: 22: 51$ & 81.24 & - & 50.96 & 1.15 & 16.70 \\
\hline E & 86.75 & 500.53 & $3: 55: 52$ & - & 18.00 & 72.31 & 1.18 & 201.79 \\
\hline
\end{tabular}

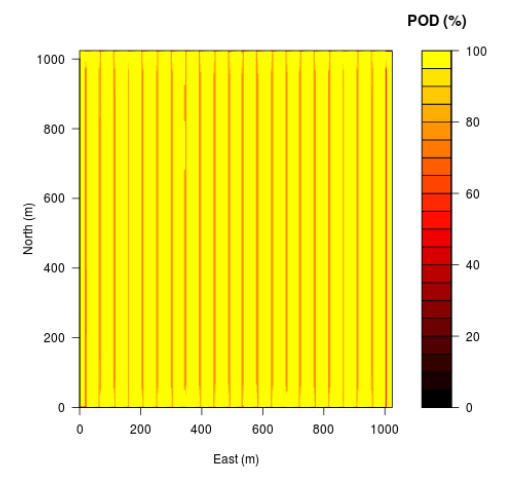

(a) 2D coverage plot of original solution.

Figure 3: Original solution obtained by the EA using a sidescan sonar.

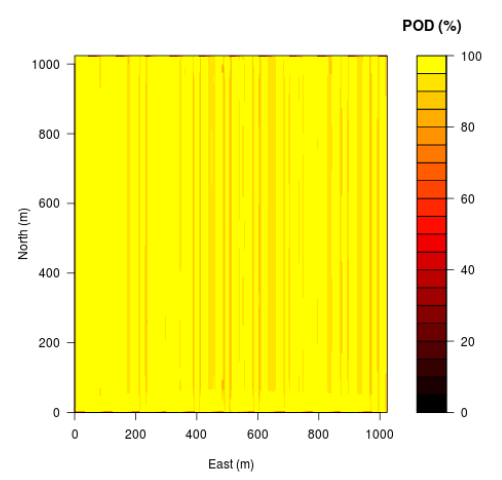

(a) 2D coverage plot of optimized solution.

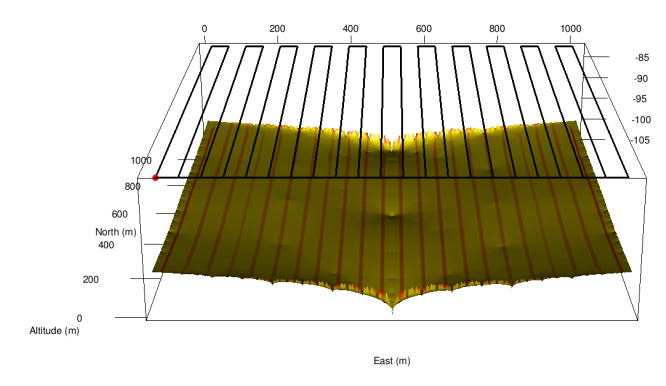

(b) 3D coverage plot of original solution.

Figure 4: 3D local optimization of the previous solution obtained by the EA using a sidescan sonar.

Table 4: Descriptive statistics of the complete set of solutions obtained for the coverage problem using a sidescan sonar.

\begin{tabular}{|l|r|r|r|}
\hline & $\Delta_{M R}(\%)$ & $\Delta_{P O D}(\%)$ & Time $(\mathrm{s})$ \\
\hline Min & -19.41 & -8.84 & 24.00 \\
\hline Mean & -4.99 & 0.59 & 64.74 \\
\hline Max & 11.81 & 5.51 & 380.00 \\
\hline SD & 5.80 & 1.76 & 67.04 \\
\hline
\end{tabular}

plementarity of both our local optimization algorithm and our EA.
Table 5: Descriptive statistics of the set of solutions excluding the ones where tracks were removed.

\begin{tabular}{|l|r|r|r|}
\hline & $\Delta_{M R}(\%)$ & $\Delta_{P O D}(\%)$ & Time (s) \\
\hline Min & -19.41 & -1.99 & 24.00 \\
\hline Mean & -5.43 & 0.81 & 64.77 \\
\hline Max & 2.09 & 5.51 & 380.00 \\
\hline SD & 5.56 & 1.28 & 68.58 \\
\hline
\end{tabular}

Similar experiments were performed, but using a multibeam instead of sidescan sonar. The improvements are considered negligible as they are smaller 
by a factor of 5 then in the previous scenario, meaning that the gains do not justify the extra processing time.

\section{CONCLUSIONS}

This paper introduced an multiobjective multi-stage approach combining EA with simulated annealing for planning minehunting operations in static 3D environment with predictable terrain. Our algorithm maintains a diverse population of feasible solutions in order to explore the search space and uses simulated annealing to improve the best solutions found and produce new solutions in the neighbourhood. Our experiments showed that the integration an ANN model to guide the search is beneficial and that the proposed local optimization phase significantly helps to improve the quality of the solutions, however at the cost of a higher computational time. We also exemplified what would be the typical output of the execution of our planning algorithm and demonstrated the role that the decision maker may have to play when planning a minehunting mission with an AUV.

In the near future we are going to explore mission planning with distinct priorities for specific areas and study mission replanning. The idea is to use these algorithms to obtain a Pareto front for each area (when replanning a mission these areas are automatically defined according to mission performance) an then to efficiently try to interconnect the coverage paths, thus becoming a variant of the travelling salesman problem.

\section{ACKNOWLEDGEMENTS}

This work is financed by the ERDF - European Regional Development Fund through the COMPETE Programme (operational programme for competitiveness) and by National Funds through the FCT - Fundação para a Ciência e a Tecnologia (Portuguese Foundation for Science and Technology) within project FCOMP-01-0124-FEDER-037281.

\section{REFERENCES}

Choset, H. (2001). Coverage for robotics - a survey of recent results. In Ann. Math. Artif. Intell. SCITEPRESS.

Darwin, C. (1859). On the Origin of Species by Means of Natural Selection, or the Preservation of Favoured Races in the Struggle for Life. John Murray, London, UK.

Deb, K. (2001). Multi-objective optimization using evolutionary algorithms. Wiley, UK.

Fang, C. and Anstee, S. (2010). Coverage path planning for harbour seabed surveys using an autonomous underwater vehicle. In OCEANS 2010 IEEE - Sydney, pages $1-8$. IEEE.

Fujimura, K. (1996). Path planning with multiple objectives. Robot Autom. Mag., 3(1):33-38.

Huang, G. (2003). Learning capability and storage capacity of two-hidden-layer feedforward networks. Neural Networks, IEEE Transactions on, 14(2):274-281.

Koopman, B. (1999). Search and Screening: General Principles with Historical Applications. The Military Operations Research Society, Virginia, USA.

Land, S. and Choset, H. (1998). Coverage path planning for landmine location. In 3 rd International Symposium on Technology and the Mine Problem.

Metropolis, N., Rosenbluth, A., Rosenbluth, M., Teller, A., and Teller, E. (1953). Equation of state calculations by fast computing machines. J. Chem. Phys., 21(6):1087-1092.

Nash, L., Hover, G., and Burns, R. (1982). Additional analyses of probability of detection (pod) in search and rescue (sar) project data. Technical report.

Stack, J. and Smith, C. (2003). Combining random and data-driven coverage planning for underwater mine detection. In OCEANS 2003. Proceedings, volume 5, pages 2463-2468 Vol.5. IEEE.

Williams, D. (2010). On optimal auv track-spacing for underwater mine detection. In Robotics and Automation (ICRA), 2010 IEEE International Conference on, pages 4755-4762. IEEE.

Xiao, J., Michalewicz, Z., Zhang, L., and Trojanowski, K. (1997). Adaptive evolutionary planner/navigator for mobile robots. Evolutionary Computation, IEEE Transactions on, 1(1):18-28.

Zitzler, E., Laumanns, M., and Thiele, L. (2001). Spea2: Improving the strength pareto evolutionary algorithm. Technical report. 